\title{
Ultra High Energy Neutrino Astronomy
}

\author{
V. Berezinsky ${ }^{a}$ \\ ${ }^{a}$ INFN - Laboratori Nazionali del Gran Sasso, I-67010 Assergi (AQ), Italy
}

The short review of theoretical aspects of ultra high energy (UHE) neutrinos and superGZK neutrinos. The sources and diffuse fluxes of UHE neutrinos are discussed. Much attention is given to comparison of the cascade and cosmic ray upper bounds for diffuse neutrino fluxes. Cosmogenic neutrinos and neutrinos from the mirror mater are considered as superGZK neutrinos.

\section{Introduction}

With Baikal and AMANDA detectors UHE neutrino astronomy entered its first stage, which will reach the decisive level with $1 \mathrm{~km}^{3}$ detector. Simultaneously, the first steps are made in building the detectors searching for superGZK neutrinos, i.e. those with energies above the GZK cutoff, $E_{\mathrm{GZK}} \sim 5 \times 10^{19} \mathrm{eV}$. Radio-telescopes, such as RICE, GLUE and FORTE are already searching for radio signal from neutrino-induced showers in ice and in the moon. Atmospheric neutrino-induced showers can be observed from the space as it is proposed in the EUSO project. Neutrino-induced horizontal atmospheric showers can be observed by the Auger array, which is now in the data-collecting phase.

Production of UHE cosmic neutrinos

occurs in $p p$ and $p \gamma$ collisions of UHE protons with the target protons/nuclei and with target low-energy photons. They can be also produced by annihilation of DM particles and by decays of superheavy particles. In all these cases neutrinos are mostly produced in the chain of pion decays, and hence neutrino astronomy is a search for locations of HE pion production.

\section{UHE neutrino sources}

can be subdivided into accelerator sources and top-down sources, where neutrinos are produced in decays and annihilation of heavy particles. The examples of such sources are given by annihilation of neutralinos in the Sun and Earth, by topological defects, which produce superheavy unstable particles, and by decays of quasi-stable super- heavy DM particles.

Usually neutrino radiation is accompanied by other types of $\mathrm{HE}$ radiations, most notably by $\mathrm{HE}$ gamma-radiation and cosmic rays $(\mathrm{CR})$. There are, however, so called "hidden sources" where all accompanying radiations are strongly or almost absorbed. The examples of such objects are the Sun and Earth, in center of which neutralinos annihilate. Another ideal example is given by mirror matter, where all mirror particles interact with visible matter gravitationally, and only mirror neutrinos can oscillate into visible ones. The almost "hidden" source is given by the Stecker model 1 of AGN, where UHE photons and protons are mostly absorbed or confined, and only HE neutrinos emerge from there. There are some other less exotic examples of astrophysical hidden sources [2].

Neutrino detection

includes four remarkable reactions:

Muon production $\nu_{\mu}+N \rightarrow \mu+$ all gives an excellent tool to search for the discrete sources, since directions of UHE muon and neutrino coincide.

Resonant production of W-boson, $\bar{\nu}_{e}+e \rightarrow$ $W^{-} \rightarrow$ hadrons results in production of monoenergetic showers with energy $E_{0}=m_{W}^{2} / 2 m_{e}=$ $6.3 \times 10^{6} \mathrm{GeV}$. This reaction has a large crosssection.

Tau production in a detector, $\nu_{\tau}+N \rightarrow \tau+$ hadrons, is characterised by time sequence of three signals: a shower from prompt hadrons, the Cherenkov light from $\tau$ and hadron shower from $\tau$-decay. SuperGZK $\nu_{\tau}$ are absorbed less in the 
Earth due to regeneration: absorbed $\nu_{\tau}$ is converted into $\tau$, which decays producing $\nu_{\tau}$ again.

Z-bursts provide a signal from the space, caused by the resonant $Z^{0}$ production on DM neutrinos, $\nu+\bar{\nu}_{\mathrm{DM}} \rightarrow Z^{0} \rightarrow$ hadrons. The energy of the detected neutrino must be tremendous:

$E_{0}=\frac{m_{Z}^{2}}{2 m_{\nu}}=1.7 \times 10^{13}\left(\frac{0.23 \mathrm{eV}}{m_{\nu}}\right) \mathrm{GeV}$.

Neutrino oscillations

play the essential role. The neutrino flavors $\bar{\nu}_{e}$ and $\nu_{\tau}$ are inefficiently produced in the accelerator sources. The flavor oscillation with probability

$$
P_{\nu_{\alpha} \rightarrow \nu_{\beta}}=\frac{1}{2} \sin ^{2} 2 \theta \sin ^{2} \frac{r}{L(E)},
$$

and oscillation length $L(E)$, given by

$L(E)=\frac{4 E}{\Delta m^{2}}=25\left(\frac{E}{10^{11} \mathrm{GeV}}\right)\left(\frac{10^{-4} \mathrm{eV}^{2}}{\Delta m^{2}}\right) \mathrm{pc}$

are very efficient due to large mixing angles $\theta$ and astrophysically very short oscillation lengths. If initial flavor ratio is given by pion decays, $\nu_{e}: \nu_{\mu}: \nu_{\tau}=1: 2: 0$, the observed flavor ratio is $\nu_{e}: \nu_{\mu}: \nu_{\tau}=1: 1: 1$ (equipartion).

HE neutrinos from early universe.

One might think (and many did think) that large neutrino fluxes can be produced at cosmological epochs with large red shift. This possibility is disfavored [3] by absorption of HE neutrinos and by nucleosynthesis bound on their fluxes. Neutrinos are absorbed in $\nu \bar{\nu}$ collisions with big-bang neutrinos and horizon of observation for neutrinos with energy $E_{\nu 0}$ (at present) is given by redshift

$$
z_{\mathrm{abs}}=7.9 \times 10^{4}\left(E_{\nu 0} / 1 \mathrm{TeV}\right)^{-1 / 3}
$$

Neutrino fluxes produced at large $\mathrm{z}$ are strongly restricted by production of $\mathrm{D}$ and ${ }^{3} \mathrm{He}$ at the epochs after Bing Bang Nucleosynthesis. Neutrinos cause e-m cascades and $\mathrm{MeV}$ photons from these cascades produce $\mathrm{D}$ and ${ }^{3} \mathrm{He}$ in collisions with ${ }^{4} \mathrm{He}$ nuclei:

$$
\begin{gathered}
\nu+\bar{\nu}_{\mathrm{DM}} \rightarrow \text { cascade } \rightarrow \mathrm{MeV} \text { photons } \rightarrow \\
\gamma_{\mathrm{MeV}}+{ }^{4} \mathrm{He} \rightarrow{ }^{3} \mathrm{He} / \mathrm{D} .
\end{gathered}
$$

\section{Accelerator sources}

Protons are assumed to be accelerated mostly by the shocks and produce neutrinos in ppcollisions in gas and in $p \gamma$ collisions in low-energy photon background.

$H E$ neutrinos from AGN cores.

This case is known as the Stecker model [1]. The massive black hole $(\mathrm{BH})$ is surrounded by the thick accretion disc. The radial flow of accreting gas to $\mathrm{BH}$ is terminated by the shock at radial distance $r_{\mathrm{sh}} \sim 10 r_{g}$. The protons are accelerated diffusively at the shock, but they cannot diffuse upstream. As a result they are dragged by gas flow downstream and are absorbed by $\mathrm{BH}$. Neutrinos are produced in $p \gamma$-collisions with UV photons of the disc radiation. HE gamma produced in the same $p \gamma$-collisions are absorbed due to $\gamma+\gamma_{\mathrm{UV}} \rightarrow e^{+}+e^{-}$. Neutrino flux from an individual AGN is not detectable, while the diffuse flux is detectable. In fact, the original prediction of the Stecker model is higher than upper limit of AMANDA, but diffuse flux in the Stecker model can be easily suppressed by the choice of parameters, in particular by increasing of $r_{\mathrm{sh}}$.

$H E$ neutrinos from AGN jets [4].

The protons are accelerated in the multiple shocks in the AGN jets, especially in their inner parts. Neutrinos are produced in the collisions with photons from the accretion disc and from photons produced in the jet by accelerated electrons and photons. Neutrino flux from individual AGN is very small, but the diffuse flux is detectable by future $1 \mathrm{~km}^{3}$ detector.

HE neutrinos from galaxy clusters [5].

The clusters of galaxies are able to keep UHE cosmic rays for a time exceeding the age of the universe. This is the key phenomenon which makes galaxy clusters the powerful sources of UHE neutrinos. The particles are accelerated in clusters by various mechanisms: in the normal galaxies by $\mathrm{SN}$ shocks, in $\mathrm{AGN}$ and $\mathrm{cD}$-galaxies, in the process of galactic merging. HE protons, confined in a cluster by intracluster magnetic fields, produce the large flux of $p p$-neutrinos due to long confinement time. The diffuse HE neutrino flux is 
determined entirely by basic parameters characterising the clusters. In particular, for the lower limit of the diffuse flux provided by normal galaxies with CR luminosity $L_{p}$ and generation index $\gamma_{g}$, both taken as ones in our galaxy, the diffuse flux is given as

$$
J_{\nu}(E) \propto L_{p} E^{-\gamma_{g}} \frac{N_{g}}{R_{\mathrm{cl}}^{3}} \xi \Omega_{b} \rho_{\mathrm{cr}},
$$

where $R_{\mathrm{cl}} \sim 2 \mathrm{Mpc}$ is the virial radius of a cluster, $N_{g} \sim 100$ is richness of a cluster, $\rho_{\text {cr }}$ is critical cosmological density, and $\xi \Omega_{b}$ is cosmological baryonic density provided by clusters.

\section{Gamma Ray Bursts.}

GRBs are most exiting sources of UHE neutrinos. There are two mechanisms of $\mathrm{HE}$ neutrino generation. In the first one 6] particles are accelerated by external shock and neutrinos are produced in $p \gamma$ collisions with GRB photons behind the shock. In the second mechanism [7] protons are accelerated by internal shocks. The shocks collide and produce the turbulence with equipartion magnetic field. In such medium the Fermi II acceleration mechanism operates, the spectrum is assumed to be $\propto 1 / E^{2}$. Neutrinos are produced in $p \gamma$ collisions with GRB photons. All estimates are very transparent and follow from assumption that the energy outputs in GRB photons, accelerated protons and produced neutrinos are about the same: $W_{\nu} \sim W_{p} \sim W_{\mathrm{GRB}}$. Then the total number of neutrinos with energy $E$ per burst is

$$
N_{\nu}(E) \sim \frac{W_{\mathrm{GRB}}}{\ln E_{\max } / E_{\min }} E^{-2},
$$

where $E_{\min }$ and $E_{\max }$ are minimum and maximum acceleration energy, respectively. Now one can write the flux of neutrinos from a single GRB, expressing it through neutrino fluence $S_{\nu}$ :

$$
F_{\nu_{\mu}+\bar{\nu}_{\mu}}(>E)=\frac{1}{3} \frac{S_{\nu}}{E \ln \left(E_{\max } / E_{\min }\right)} .
$$

For $S_{\nu} \leq S_{\mathrm{GRB}}^{\max }=1 \times 10^{-4} \mathrm{erg} / \mathrm{cm}^{2}$, the number of muons per GRB burst in ICECUBE is $N_{\mu}<$ 0.1 for $E_{\mu} \geq 1 \mathrm{TeV}$.

The diffuse flux is estimated in identical way through the local neutrino emissivity $\mathcal{L}_{\nu}(0)$ and evolutionary factor $k_{\text {evol }}$

$$
J_{\nu_{\mu}+\bar{\nu}_{\mu}}(E)=\frac{1}{3} \frac{c H_{0}^{-1}}{4 \pi} \frac{\mathcal{L}_{\nu}(0)}{E^{2} \ln E_{\max } / E_{\min }} k_{\mathrm{evol}},
$$

where evolutionary factor is given by

$$
k_{\mathrm{evol}}=\int_{0}^{z_{\max }} \frac{d z}{(1+z)^{2}} \frac{f_{\mathrm{evol}}(z)}{\sqrt{(1+z)^{3} \Omega_{m}+\Lambda}} .
$$

For the evolutionary function $f_{\text {evol }}(z)$ we take the case of strong star formation evolution from [8], which results in $k_{\text {evol }}=7.0$ as a maximum value. For the emissivity we use

$$
\mathcal{L}_{\nu}(0) \leq \mathcal{L}_{\mathrm{GRB}}(0)=0.6 \times 10^{43} \mathrm{erg} / \mathrm{Mpc}^{3} \mathrm{yr},
$$

where the local GRB emissivity is taken from 9]. As a result we obtain $E^{2} J_{\nu_{\mu}+\bar{\nu}_{\mu}}(E)=3.9 \times$ $10^{-10} \mathrm{GeV} / \mathrm{cm}^{2} \mathrm{~s}$ sr, i.e. one order a magnitude lower than in 7] (see Fig. 2]) (note that in 7] the emissivity is by factor of 3 larger). Diffuse neutrino flux from GRBs can be only marginally detected by ICECUBE, but time and position correlations with GRBs make reliable even 1 - 3 detected events.

\section{Non-accelerator neutrino sources}

These sources include objects with annihilation of DM (the Sun, Earth, cores of the galaxies), objects with the decays of superheavy DM particles (galactic halos) and topological defects. In the last two cases neutrinos are produced in the decays of superheavy particles with the masses up to $M_{\mathrm{GUT}} \sim 10^{16} \mathrm{GeV}$. A particle decays to virtual particles, partons, which are cascading due to QCD interaction, and at the confinement radius cascade partons are converted to hadrons, most of which are pions. Neutrinos are produced in pion decays with spectrum which can be approximately described at highest energies as $d E / E^{2}$.

Neutralino annihilation in the Sun and Earth.

Neutralino is the best motivated DM particle. Crossing the Sun or Earth neutralino can loose its energy in collisions with nuclei and diminish its velocity below the escape velocity. If it happens, the neutralino is gravitationally trapped in 


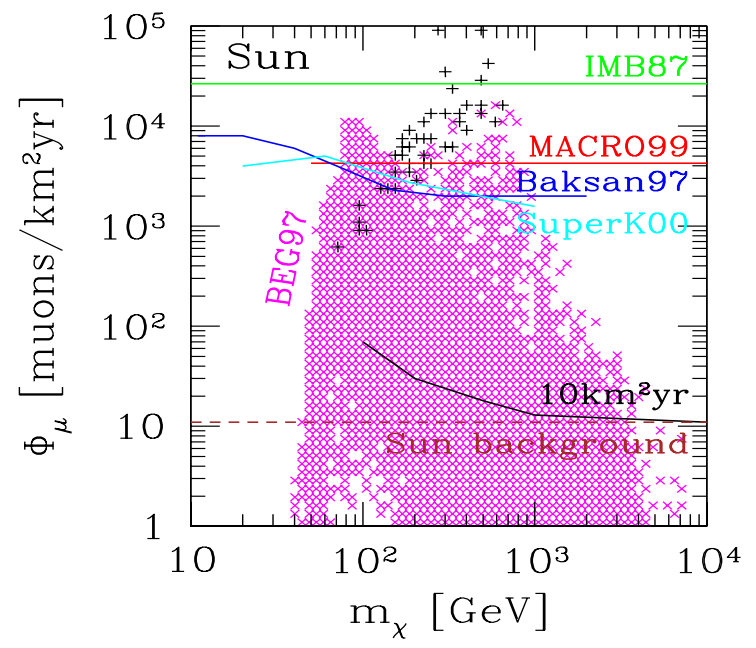

Figure 1. Underground muon flux produced by neutralino annihilation in the Sun, from ref. 10]. The points correspond to different supersymmetric parameters, $m_{\chi}$ is the neutralino mass. The upper curves give the upper limits from various experiments. Curve "Sun background" corresponds to the neutrino flux induced by $\mathrm{CR}$ interacting with the Sun matter. The region above the curve $10 \mathrm{~km}^{2} \mathrm{yr}$ is accessible for a detector with the given exposure, e.g for the ICECUBE detector.

the object, and loosing further their energies, neutralinos are accumulated in the center of a celestial body. Annihilating there they produce pions and then neutrinos. The process of annihilation strongly depends on neutralino mass and composition (mixture of basic fields: zino, bino and two higgsinos). Fig. 1] shows the neutrinoinduced underground muon flux from annihilation of neutralinos in the Sun. The Sun background is caused by neutrinos produced by cosmic rays bombarding the Sun.

Topological defects (TDs) are produced in early universe due to symmetry breaking accompanied by the phase transitions. In many cases TDs become unstable and decompose to constituent fields, superheavy gauge and Higgs bosons (Xparticles), which then decay producing UHE neutrinos (see [1], and 12] for a review)

Monopoles (M) are produced due to $G \rightarrow H \times$ $U(1)$ symmetry breaking. $M \bar{M}$ annihilation can produce QCD cascade and neutrinos. Scenarios with the free monopole annihilation and with the bound $M \bar{M}$ systems (monopolonia) are disfavored for production of large neutrino fluxes.

Ordinary strings are produced by $U(1)$ symmetry breaking. There are several mechanisms by which ordinary strings can produce HE neutrinos: collapse of the string loops, self-intersection, annihilation of cusps, production and annihilation of tiny loops. In most cases produced neutrino fluxes are too small for detection.

Superconducting strings can be powerful sources of neutrinos. In a wide class of elementary particle models strings behave like superconducting wires. Moving though cosmic magnetic fields, such strings develop electric current. Superconducting strings produce superheavy X-particles, when electric current in the string reaches the critical value. X-particles escape from a string and decay. This process is strongly enhanced near the cusps, the peculiar points on a string, where the velocity of an oscillating string reaches the speed of light. The cusp emission of superheavy X-particles provides a realistic source of UHE neutrinos.

Monopoles connected by strings are produced in the $G \rightarrow H \times U(1) \rightarrow H \times Z_{n}$ sequence of symmetry breaking. At the first symmetry breaking monopoles are produced, at the second one each monopole get attached to $n$ strings. Monopoles oscillate near the equilibrium positions, generating UHE gauge bosons with neutrinos produced at their decays.

Necklaces correspond to the case above when $n=2$, i.e. when each monopole is attached to two strings, and a loop reminds a necklace with monopoles playing the role of beads. In the process of evolution the strings shrink due to gravitational radiation and $M \bar{M}$ pairs in the necklace inevitably annihilate. This model is most plausible and well developed for UHE neutrino production.

\section{Diffuse fluxes and upper limits}

In this section I will discuss the cascade and cosmic ray upper limits, and compare them with the model calculations of diffuse fluxes from var- 
ious astrophysical sources.

\subsection{Cascade upper limit}

The cascade upper limit on HE and UHE neutrino fluxes 132 is provided due to e-m cascades initiated by $\mathrm{HE}$ photons or electrons which always accompany production of neutrinos. Colliding with the target low-energy photons, a primary photon or electron produce e-m cascade due to reactions $\gamma+\gamma_{\text {tar }} \rightarrow e^{+}+e^{-}, e+\gamma_{\text {tar }} \rightarrow e^{\prime}+\gamma^{\prime}$, etc. The standard case is given by production of HE neutrinos in extragalactic space, and the cascade develops due to collisions with CMB photons $\left(\gamma_{\mathrm{tar}}=\gamma_{\mathrm{CMB}}\right)$. In case neutrino production occurs in a galaxy, the accompanying photon can either freely escapes from a galaxy and produce cascade in extragalactic space, or produce cascade on the background radiation (e.g. infra-red) inside the galaxy. In the latter case the galaxy should be transparent for the cascade photons in the range $10 \mathrm{MeV}$ - $100 \mathrm{GeV}$.

The spectrum of the cascade photons is calculated 132]: in low energy part it is $\propto E^{-3 / 2}$, at high energies $\propto E^{-2}$ with a cutoff at some energy $\epsilon_{\gamma}$. The energy of transition between two regimes is given approximately by $\epsilon_{c} \approx\left(\epsilon_{t} / 3\right)\left(\epsilon_{\gamma} / m_{e}\right)^{2}$, where $\epsilon_{t}$ is the mean energy of the target photon. In case the cascade develops in extragalactic space $\epsilon_{t}=6.35 \times 10^{-4} \mathrm{eV}, \epsilon_{\gamma} \sim 100 \mathrm{GeV}$ (absorption on optical radiation), and $\epsilon_{c} \sim 8 \mathrm{MeV}$. The cascade spectrum is very close to the EGRET observations in the range $3 \mathrm{MeV}$ - $100 \mathrm{GeV}$ [14. The observed energy density in this range is $\omega_{\text {EGRET }} \approx(2-3) \times 10^{-6} \mathrm{eV} / \mathrm{cm}^{3}$. The upper limit on $\mathrm{HE}$ neutrino flux $J_{\nu}(>E$ ) (sum of all flavors) is given by chain of the following inequalities

$$
\begin{gathered}
\omega_{\text {cas }}>\frac{4 \pi}{c} \int_{E}^{\infty} E J_{\nu}(E) d E>\frac{4 \pi}{c} E \int_{E}^{\infty} J_{\nu}(E) d E \equiv \\
\frac{4 \pi}{c} E J_{\nu}(>E),
\end{gathered}
$$

which in terms of the differential neutrino spec$\operatorname{trum} J_{\nu}(E)$ gives

$E^{2} J_{\nu}(E)<\frac{c}{4 \pi} \omega_{\text {cas }}$, with $\omega_{\text {cas }}<\omega_{\text {EGRET }}$

Eq. (2) gives the rigorous upper limit on the neutrino flux. It is valid for neutrino production

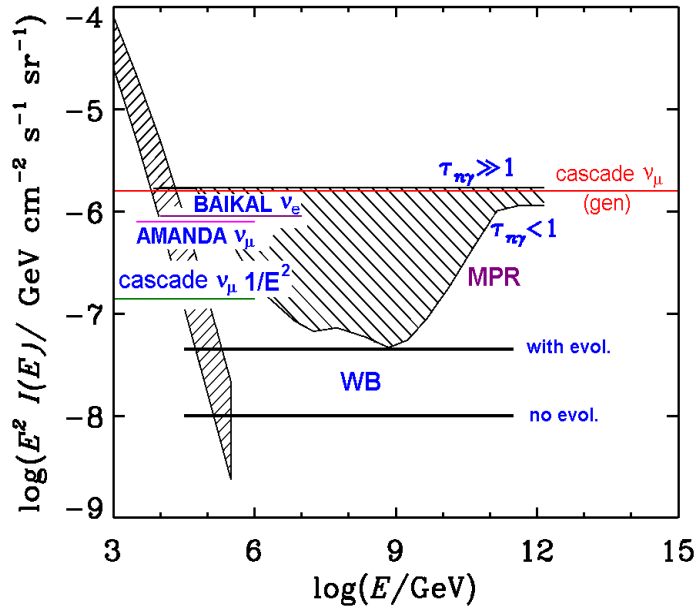

Figure 2. Cascade and CR upper limits. The general cascade upper limit (2) is shown by line "cascade $\nu_{\mu}$ " with additional factor $1 / 3, E^{-2}$ cascade upper limit is given by line "cascade $\nu_{\mu} 1 / E^{2}$ ". "BAIKAL $\nu_{e}$ " and "AMANDA $\nu_{\mu}$ " are for Baikal and AMANDA upper limits, respectively. The Waxman-Bahcall upper limits are given by WB lines with and without evolution. Mannheim-Protheroe-Rachen limits are marked by MPR with the optical depth for neutrons: $\tau_{n \gamma} \gg 1$ and $\tau_{n \gamma}<1$. Atmospheric neutrinos are shown by the hatched diagonal strip.

by HE protons, by TDs, by annihilation and decays of superheavy particles, i.e. in all cases when neutrinos are produced through decay of pions and kaons. It is valid for production of neutrinos in extragalactic space and in galaxies, if they are transparent for the cascade photons. It holds for arbitrary neutrino spectrum falling down with energy. If one assumes some specific shape of neutrino spectrum, the cascade limit becomes stronger. For $E^{-2}$ generation spectrum one immediately obtains

$E^{2} J_{i}(E) \leq \frac{1}{3} \frac{c}{4 \pi} \frac{\omega_{\text {cas }}}{\ln \left(E_{\max } / E_{\min }\right)}$,

where $i=\nu_{\mu}+\bar{\nu}_{\mu}$ or $i=\nu_{e}+\bar{\nu}_{e}$.

AMANDA and Baikal collaborations in fact assume $E^{-2}$ neutrino spectrum to obtain observational upper limits. In Fig. 2 we plot both the 
general cascade upper limit (2) and $E^{-2}$ cascade upper limit (3), both for $\nu_{\mu}+\bar{\nu}_{\mu}$ neutrino flavors. For the $E^{-2}$ cascade upper limit, the AMANDA-B10 parameters $E_{\min }=6 \mathrm{TeV}$ and $E_{\max }=1000 \mathrm{TeV}$ [15] are used. Baikal upper limit is taken from [16.

AMANDA and Baikal did not reach yet the cascade upper limit (see Fig. 2).

\subsection{Cosmic Ray (CR) upper limits}

Cosmic rays leaking out of HE neutrino sources should not exceed the observed flux: this is the essence of CR upper limits on the neutrino flux.

The Waxman-Bahcall upper limit [17].

The neutrino sources which produce UHECR with energies $10^{19}-10^{21} \mathrm{eV}$ are considered. It is assumed that neutrinos are produced by protons in $p \gamma$ collisions in a source, and that protons are loosing the fraction of energy $\epsilon<1$. The generation spectrum is assumed $\propto 1 / E^{2}$. Escaping protons should not exceed the observed flux of UHECR and it imposes the upper limit on neutrino flux. These upper limits are plotted in Fig. 2] by the WB lines for evolutionary and non-evolutionary cases.

Note, that this limit is not valid for sources with maximum energy of accelerated protons $E_{\max } \leq 1 \times 10^{19} \mathrm{eV}$, for all sources where protons are confined (e.g. clusters of galaxies and the AGN core models [1]), and for all non-accelerator sources discussed above.

The Mannheim-Protheroe-Rachen upper limits.

The CR upper limit can be relaxed taking into account the absorption of HE protons in a source. In the work [18 the exit of protons from a source is provided by production and escape of the neutrons with their consequent decays to the protons. This upper limit is constructed mainly for AGN, assuming various optical depth $\tau_{n \gamma}$ for escaping protons. In contrast to WB limit, the maximum energy of accelerated protons is allowed in a wide range $10^{6}-3 \times 10^{13} \mathrm{GeV}$. The upper bound on extragalactic proton flux for this energy interval is extracted from observations. The spectrum of accelerated protons is assumed $\propto 1 / E^{2}$, but the resulting proton spectrum at observation is evaluated. In Fig. 2 the MPR upper limits are

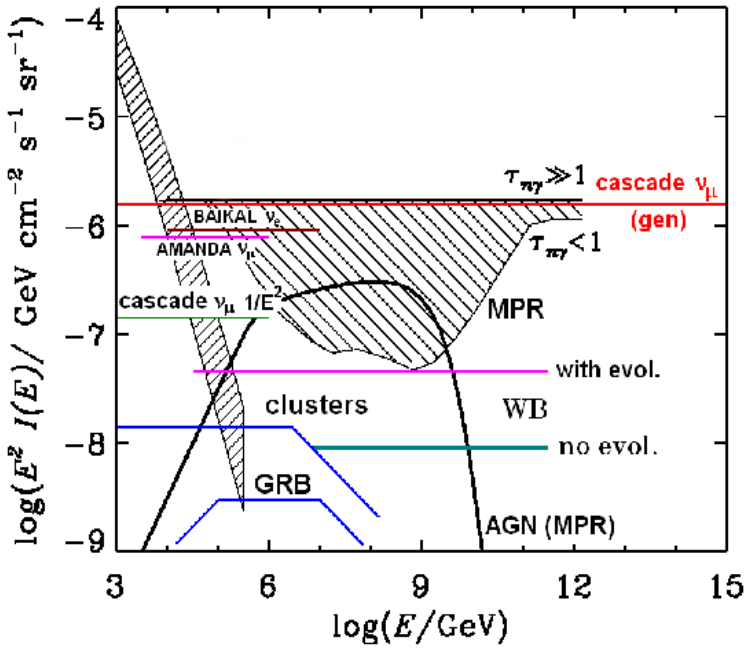

Figure 3. Diffuse fluxes from AGN jets, GRBs and galaxy clusters, compared with upper bounds.

shown for two extreme cases $\tau_{n \gamma}<1$ (neutron transparent sources) and $\tau_{n \gamma} \gg 1$ (neutron nontransparent sources).

CR upper limits are valid for AGN and GRBs. They are not valid for TDs, annihilation and decay of DM particles and hidden sources [19. They are also not valid for such conservative accelerator sources as galaxy clusters. Such long list of exclusions contradicts the standard definition of upper limit in physics. It deserves more the name landmark for future HE neutrino detectors, especially in the case of the WB limit.

In Fig. 3 diffuse fluxes from AGN jets, GRBs and galaxy clusters are shown in comparison with cascade upper limits and CR benchmarks. Note, that diffuse flux from galaxy clusters (the case without evolution) exceeds the corresponding WB limit.

\section{SuperGZK neutrinos}

Soon after prediction of the GZK cutoff, it was noticed that this phenomenon is accompanied by a flux of extremely high energy neutrinos, which in some models exceeds the flux of parent protons [20]. For detection of these neutrinos the horizontal EAS have been proposed [13]. The boost to this field has been recently given by the projects 


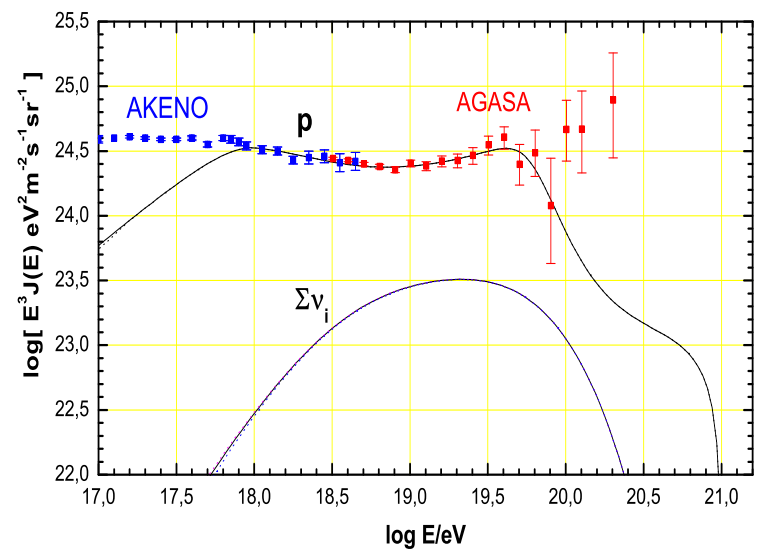

Figure 4. Diffuse proton and cosmogenic neutrino fluxes in the AGN model [27, without cosmological evolution and with $E_{\max }=1 \times 10^{21} \mathrm{eV}$. The generation spectrum index is taken $\gamma_{g}=2.7$ to provide the best fit of the Akeno-AGASA data by the proton spectrum (curve $\mathrm{p}$ ).

of observation of superGZK neutrinos from the space 21] and by the first results of observation of radio signal from the neutrino-induced cascades in the ice [22] and in the Moon 23]. In all these detectors the threshold is very high, and these methods are mostly effective for superGZK neutrinos with energies $E \gtrsim 1 \times 10^{20} \mathrm{eV}$.

In what sources these tremendous energies are possible?

The accelerator sources are disfavored, if one limits himself by the shock acceleration. Neutrino energy $E_{\nu}>1 \times 10^{20} \mathrm{eV}$ implies proton energy $E_{p} \sim 20 E_{\nu}>2 \times 10^{21} \mathrm{eV}$. Such energy is only marginally possible for non-relativistic shocks in AGN jets, and it is too high for ultrarelativistic shocks 24. However, there are many proposals of plasma mechanisms of acceleration with $E_{\max }$ much higher than $1 \times 10^{21} \mathrm{eV}$, see e.g. 25. One may optimistically expect that $E_{\max } \sim 1 \times 10^{22} \mathrm{eV}$ is not the excluded possibility.

TDs and SHDM particles naturally provides very high energies up to $E_{\mathrm{GUT}} \sim 10^{16} \mathrm{GeV}$.

\subsection{Cosmogenic neutrinos}

These neutrinos are produced by UHECR interacting with CMB: $p+\gamma_{\mathrm{CMB}} \rightarrow \pi^{ \pm} \rightarrow$ neutrinos. The cosmogenic neutrino flux can

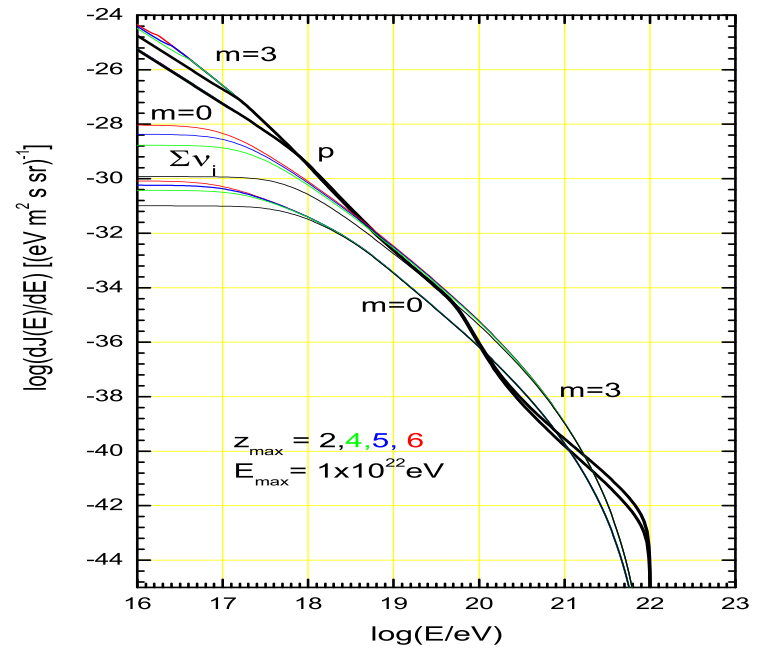

Figure 5. Diffuse cosmogenic neutrino fluxes in the AGN model 27] with and without evolution. The evolution is described by factor $(1+z)^{m}$ up $z_{\max }$. The maximal energy is $E_{\max }=1 \times 10^{22} \mathrm{eV}$ in all cases. The non-evolutionary case is shown by the curves $m=0$. The proton spectrum, given by curve $\mathrm{p}$, fits well all observations.

be approximately evaluated using the unmodified CR flux $J_{p}^{\mathrm{unm}}(E)$, i.e. one where only adiabatic energy losses (red shift) are taken into account. Neutrino flux at $z=0$ is given by 20 .

$J_{\nu}(E)=\frac{2}{3} 3\left(\frac{E_{\nu}}{E_{p}}\right)^{\gamma_{g}-1} \frac{1}{1-\alpha^{\gamma_{g}-1}} J_{p}^{\mathrm{unm}}(E)$,

where $2 / 3$ is a fraction of charged pions produced by very high energy proton, $E_{\nu} / E_{p} \approx 0.05$ is a fraction of proton energy transferred to neutrino, and $\alpha$ is a fraction of energy lost by proton in $p \gamma$ collision ( $\alpha$ varies from 0.22 in $\Delta$-resonance to 0.5 at extremely high energies). The term with $\alpha$ in Eq. (44) takes into account the multiple collisions of proton with CMB photons, especially important in the evolutionary case. At low energies neutrino flux becomes flat. In evolutionary models the unmodified proton flux and neutrino flux can be much higher than UHECR flux calculated with all energy losses included. The recent calculations of cosmogenic neutrinos [26] use the normalization to the observed UHECR flux. The neutrino fluxes are small in non-evolutionary models with $E_{\max } \leq 1 \times 10^{21} \mathrm{eV}$ and large in the 


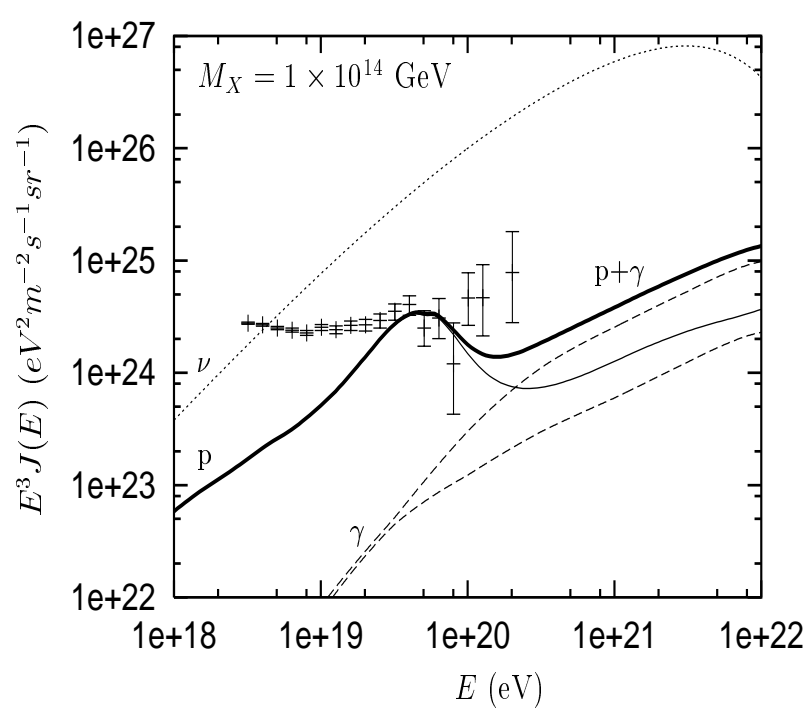

Figure 6. Diffuse neutrino spectrum from necklaces for $M_{X}=1 \times 10^{14} \mathrm{GeV}$ 30]. The thick curve gives $p+\gamma$ flux normalized to the AGASA data.

models with strong cosmological evolutions and with $E_{\max } \geq 1 \times 10^{22} \mathrm{eV}$. In Fig. 4 we present the neutrino flux produced in the non-evolutionary AGN model [27] with $E_{\max }=1 \times 10^{21} \mathrm{eV}$ and with generation spectrum index $\gamma_{g}=2.7$. This model describes well the UHECR spectra observed by all existing detectors, except the highest energy AGASA events (see Fig. (4). The calculated neutrino spectrum [28] gives the low limit on the cosmogenic neutrino flux imposed by the observed flux of UHECR and assumption $E_{\max } \leq 1 \times 10^{21} \mathrm{eV}$. This flux is smaller than the low limit found in the paper by Fodor et al 26] and is essentially undetectable. The large neutrino fluxes produced in the AGN models with $E_{\max } \leq 1 \times 10^{22} \mathrm{eV}$ are shown in Fig. 15 28. The calculated UHECR flux in all cases fits well the observational data. Both the models with cosmological evolution $(1+z)^{m}$ up to $z_{\max }$, and non-evolutionary models with $\mathrm{m}=0$ were used. One can notice that evolutionary models predicts much larger neutrino fluxes. They are detectable by future arrays.

In case the UHECR primaries are extragalactic nuclei, the flux of superGZK neutrinos is suppressed. The nuclei responsible for superGZK neutrinos are promptly photo-disintegrated to A nucleons, which produce neutrinos in photopion collisions. Thus, the energy of neutrino is $E_{\nu} \sim(0.05 / A) E_{N}$, where $E_{N}$ is an energy of nucleus. Putting $E_{\nu} / E_{N}$ ratio in Eq. (4), and taking into account $3 \mathrm{~A}$ neutrinos produced by one nucleus, we obtain the additional suppression of ratio $J_{\nu}(E) / J_{N}^{\text {unm }}(E)$ by factor $A^{2-\gamma_{g}}$. Besides, the energy of accelerated nucleus should be $E_{N}>20 A \times 10^{20} \mathrm{eV}$, e.g. $1.1 \times 10^{22} \mathrm{eV}$ in case of iron.

The extragalactic nuclei as UHECR primaries have another interesting aspect connected with neutrinos of lower energies $E_{\nu} \lesssim 10^{15} \mathrm{eV}$. In this energy region the neutrino production is dominated by neutron decays with neutrons produced in photodissociation of UHE nuclei 29].

\subsection{Topological defects}

TDs as neutrino sources are described in Section 3 Neutrino fluxes from TDs are limited by the cascade upper limit (2). Usually it excludes too large masses of X-particles, in decays of which neutrinos are produced. The diffuse neutrino flux from necklaces has been calculated in [30. The neutrino spectrum is shown in Fig. [6] for $M_{X}=1 \times 10^{14} \mathrm{GeV}$, allowed by the cascade upper limit (see Fig. 17). The spectrum is normalized to fit the AGASA data with help of protons and photons ( $p+\gamma$ curve). One can see that in superGZK region the neutrino flux is two orders of magnitude higher than $p+\gamma$ flux. This model provide much higher neutrino energies than that of cosmogenic neutrinos and much higher fluxes.

\subsection{Mirror neutrinos}

Mirror matter can be most powerful source of superGZK neutrinos not limited by the usual cascade limit 34.

Existence of mirror matter is based on the deep theoretical concept, which was introduced by Lee and Yang [31], Landau [32] and most notably by Kobzarev, Okun and Pomeranchuk 33. Particle space is a representation of the Poincare group. Since the space reflection $\vec{x} \rightarrow-\vec{x}$ and time shift $t \rightarrow t+\Delta t$ commute as the coordinate transformations, the corresponding inversion operator $I_{s}$ and the Hamiltonian $H$ must commute, too: 


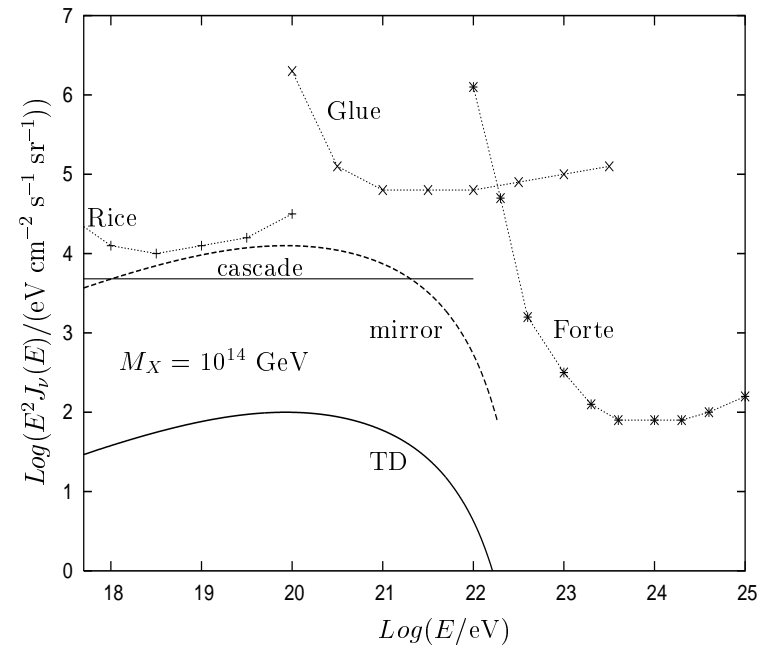

Figure 7. Diffuse flux of the visible neutrinos from mirror necklaces with $M_{X}=1 \times 10^{14} \mathrm{GeV}$. The flux is limited by observations of RICE, GLUE and FORTE. Note, that neutrino flux exceeds the general cascade upper limit (2). TD curve gives the flux from the ordinary necklaces.

$\left[I_{s}, H\right]=0$. Because the parity operator $P$ does not commute with $H$ (i.e. parity is not conserved) Lee and Yang suggested that $I_{s}=P \cdot R$, where the operator $R$ generates the mirror particle space, and thus $I_{s}$ transfers the left states of ordinary particles into right states of the mirror particles and vise versa. In fact, the assumption of Landau is similar: one may say that he assumed $R=C$.

The mirror particles have interactions identical to the ordinary particles, but these two sectors interact with each other only gravitationally [33. Gravitational interaction mixes the visible and mirror neutrino states, and thus causes the oscillation between them. A cosmological scenario must provide the suppression of the mirror matter and in particular the density of mirror neutrinos at the epoch of nucleosynthesis. It can be obtained in the two-inflaton model 34 . The rolling of two inlatons to minimum of the potential is not synchronised, and when the mirror inflaton reaches minimum, the ordinary inflaton continues its rolling, inflating thus the mirror matter produced by the mirror inflaton. While mirror matter density is suppressed, the mirror topological defects can strongly dominate 34].
Mirror TDs copiously produce mirror neutrinos with extremely high energies typical for TDs, and they are not accompanied by any visible particles. Therefore, the upper limits on HE mirror neutrinos in our world do not exist. All HE mirror particles produced by mirror TDs are sterile for us, interacting with ordinary matter only gravitationally, and only mirror neutrinos can be efficiently converted into ordinary ones due to oscillations. The only (weak) upper limit comes from the resonant interaction of converted neutrinos with DM neutrinos: $\nu+\bar{\nu}_{\mathrm{DM}} \rightarrow Z^{0}$ 34. The strongest limit on the fluxes of superGZK neutrinos are given by radio observations [22] and 23]. In Fig. [7] we present the flux of visible neutrinos from the mirror necklaces with $M_{X}=1 \times 10^{14} \mathrm{GeV}$, limited by radio observations.

\section{Conclusions}

High energy neutrino astronomy with the future ice/underwater $1 \mathrm{~km}^{3}$ detectors will reach the decisive stage in searching for neutrino sources and diffuse fluxes.

At present with AMANDA and Baikal the most rigorous cascade upper limit on the diffuse flux is almost reached. To demonstrate existence of this limit is an important goal, which will prove the absence of exotic physics such as e.g. large diffuse fluxes from the hidden sources.

Detection of accelerator sources (and/or diffuse fluxes from them) such as AGN and GRBs will clarify the nature of these sources and acceleration mechanisms.

Detection of non-accelerator sources might result in discovery of "new" physics: (i) Supersymmetry and Dark matter in case of HE neutrinos from the Sun and Earth, (ii) Topological Defects (diffuse flux is higher than $C R$ upper limits or presence of neutrinos with energies $E_{\nu}>10^{21}-10^{22} \mathrm{eV}$ ), and (iii) Mirror Matter (diffuse flux is higher than cascade upper limit.

Detection of superGZK neutrinos will be the real break-through either in acceleration physics or in physics beyond the Standard Model (Topological Defects, Superheavy Dark Matter, Mirror Matter). 


\section{REFERENCES}

1. F.W. Stecker et al, Phys. Rev. Lett., 66, 2697 (1991); F.W. Stecker and M.H. Salamon, Sp. Sci. Rev. 75, 341 (1996).

2. V. S. Berezinsky, S. V. Bulanov, V. A. Dogiel, V. L. Ginzburg and V. S. Ptuskin, Astrophysics of Cosmic Rays, North-Holland 1990.

3. V. Berezinsky, Nucl. Phys. B 380, 478 (1992); R. Protheroe, T. Stanev, V. Berezinsky, Phys. Rev. D 51, 4134 (1995).

4. K.Mannheim, Astropart. Phys. 3, 295 (1995); A. Atoyan and C.D. Dermer, Ap. J. 586, 79 (2003);

A. Atoyan and C.D. Dermer, New Astron. Rev. 48, 381 (2004).

5. V. Berezinsky, P. Blasi, V. Ptuskin, Aastroph. J. 487, 529 (1997);

P. Blasi and S. Colafrancesco, Astropart. Phys. 122, 169 (1999);

S. Gabici and P. Blasi, Astrophys. J. 583, 695 (2003).

6. M. Vietri, Phys. Rev. Lett. 80, 3690 (1998).

7. E. Waxman and J. Bahcall, Phys. Rev. Lett. 78, 2292 (1997).

8. S.D. Wick, C.D. Dermer, A.Atoyan, Astropart. Phys. 21, 125 (2004).

9. Maarten Schmidt, Astroph. J. 559, L79 (2001) and private communication 2002.

10. P. Gondolo, astro-ph/0403064

11. C.T. Hill, D.N. Schramm, T.P. Walker, Phys. Rev. D 36. 1007 (1987).

12. V. Berezinsky, Nucl. Phys. B (Proc. Suppl) 75A, 119 (1999).

13. V. Berezinsky and A. Smirnov, Ap. Sp. Sci., 32, 461 (1975).

14. P. Sreekumar [EGRET collaboration], Astroph. J. 494, 523 (1998).

15. J. Ahrens et al. [AMANDA collaboration], Phys. Rev. Lett. 90, 251101 (2003).

16. C. Spiering (for the Baikal collaboration), astro-ph/0404096

R.Wischnewski (for the Baikal collaboration), astro-ph/0305302

17. E. Waxman and J. Bahcall, Phys. Rev. D 59, 023002 (1998).

18. K. Manheim, R.J. Protheroe, J.P. Rachen, Phys. Rev. D 63, 023003 (2000), astro-ph/9812398.

19. J. Bahcall and E. Waxman, Phys. Rev. D 64, 023002 (2001).

20. V.S. Berezinsky and G.T. Zatsepin, Phys. Lett. B 28, 423 (1969) and Soviet Journal of Nucl. Phys. 11, 111 (1970).

21. see http://www.euso-misson.org/ (EUSO); http://heawww.gsfc.nasa.gov/docs/gamcosray /hecr/OWL/ (OWL).

22. N.G. Lehtinen et al., [FORTE collaboration], Phys. Rev. D 69, 013008 (2004);

I. Kravchenko, et al., [RICE collaboration], astro-ph/0306408

23. P. W. Gorham et al., [GLUE collaboration], Phys. Rev. Lett. 93, 041101 (2004).

24. Y.A. Gallant and A. Achterberg, MNRAS, 305, L6 (1999).

25. V.P. Vlasov, S.K. Zdanov, B.A. Trubnikov, Soviet Plasma Physics, 16, 1457 (1990);

P. Chen, T. Tajima, Y. Takahashi, Phys. Rev. Lett. 89, 161101 (2002).

26. R. Engel, D. Seckel, T. Stanev, Phys. Rev. D 64, 093010 (2001);

O. Kalashev, V. Kuzmin, D. Semikoz, G. Sigl, Phys. Rev. D 66, 063004 (2002);

Z. Fodor, S.D. Katz, A. Ringwald, H. Tu, JCAP 0311, 015 (2003).

27. V. Berezinsky, A. Gazizov, S. Grigorieva, hep-ph/0204357 and astro-ph/0410650

28. V. Berezinsky, A. Gazizov, S. Grigorieva, in preparation.

29. D. Hooper, A. Taylor, S. Sarkar, astro-ph/0407618

M. Ave, N. Busca, A. V. Olinto, A. A. Watson, T. Yamamoto, astro-ph/0409316 (presented at CRIS 04, Catania, May 2004).

30. R. Aloisio, V. Berezinsky and M. Kachelriess, Phys. Rev. D 69, 094023 (2004).

31. T. D. Lee and C. N. Yang, Phys. Rev. 104, 254 (1956).

32. L.D. Landau, JETP 32, 405 (1957).

33. I.Yu. Kobzarev, L.B. Okun, and I.Ya. Pomeranchuk, Sov. J. Nucl. Phys. 3, 837 (1966).

34. V.Berezinsky and A.Vilenkin, Phys. Rev. D 62, 083512 (2000). 